According to our findings, abnormal uterine bleeding is considered the guiding symptom for the diagnosis of this oncological pathology, being to one of the most frequent reasons to demand a gynecological evaluation.

Endometrial polyps are the main observed lesions in our cohort, in both ultrasound exam and hysteroscopy.

\section{LSR PROMOTES TUMOR PROGRESSION BY REGULATING SIGNAL TRANSDUCTION OF APOPTOSIS AND FERROPTOSIS IN ENDOMETRIAL CANCER}

${ }^{1} Y$ Nagase* $^{*}{ }^{1} \mathrm{~K}$ Hiramatsu, ${ }^{2} \mathrm{M}$ Funauchi, ${ }^{1} \mathrm{~S}$ Nakagawa, ${ }^{1} \mathrm{~S}$ Matsuzaki, ${ }^{1} \mathrm{E}$ Kobayashi, ${ }^{1} \mathrm{~T}$ Kimura, ${ }^{2} \mathrm{~S}$ Serada, ${ }^{1} \mathrm{Y}$ Ueda, ${ }^{2} \mathrm{~T}$ Naka, ${ }^{1} \mathrm{~T}$ Kimura. ${ }^{1}$ Osaka University Graduate School of Medicine, Department of Obstetrics and Gynecology, Osaka, Japan; '2/wate Medical University, Institute for Biomedical Sciences Molecular Pathophysiology

\subsection{6/ijgc-2021-ESG0.144}

Introduction/Background* Since advanced endometrial cancer (EC) remains a disease with a poor prognosis, the development of novel therapeutic agents are warranted. Previously, we identified lipolysis-stimulated lipoprotein receptor (LSR) as a highly expressed molecule in ovarian cancer (OC) cells and developed an anti-LSR monoclonal antibody. The antibody significantly suppressed tumor growth in EC as well as OC, however the mechanism is largely unclear, and the function of LSR in cancer cells needs to be elucidated. In this study, we focused on apoptosis and ferroptosis in programmed cell deaths and investigated the function of LSR using in vitro and bioinformatic analysis.

Methodology We evaluated LSR expression by immunohistochemistry and analyzed overall survival (OS) and clinicopathological features in 228 EC patients. To investigate the mechanism by which LSR affects the prognosis of EC patients, the pathway enrichment analysis was conducted using published proteomic data of EC. In vitro analyses were performed using two human EC cell lines (HEC1 and HEC116) and the activity of signaling pathways were examined by western blotting.

Result(s)* Patients were divided into two groups based on LSR expression; High (strongly stained in $\geq 25 \%$ of the lesion, $\mathrm{n}=153$ ) and Low (strongly stained in $<25 \%$ of the lesion, $\mathrm{n}=75$ ) groups. 5 -year OS rate in High group was significantly lower than Low group (hazard ratio: 3.53, 95\% confidence interval: $1.35-9.24, \mathrm{p}=0.01)$. The pathway analysis demonstrated that proteins correlated with high LSR expression were enriched in MAPK signaling pathway, glutathione metabolism, and cysteine and methionine metabolism. In vitro and western blot analyses showed that LSR-knockdown suppressed EC cell proliferation and the phosphorylation of MEK/ERK signaling pathway including MEK1/2, ERK1/2, and p90RSK. ERK1/2knockdown also suppressed cell proliferation, suggesting that LSR contributed to EC cell proliferation through the MEK/ ERK pathway, which is one of the apoptotic signaling pathway. In addition, LSR-knockdown suppressed the expression of cystine/glutamate antiporter (xCT) and GPX4, which inhibit ferroptosis by regulating cystine/glutamine metabolism, as determined by western blot analysis.

Conclusion* LSR contributes to tumor progression and poor prognosis by regulating apoptotic and ferroptotic signaling pathways in endometrial cancer. LSR may be a novel therapeutic target molecule in EC.

\section{COMPARING CHARACTERISTICS OF ENDOMETRIAL CANCER IN SOUTH ASIAN AND WHITE ETHNICITY WOMEN IN ENGLAND}

1:25 Mohammed, 1;3 K Polymeros, ${ }^{3} \mathrm{R}$ Wickham-Joseph, ${ }^{3} \mathrm{I}$ Luqman, ${ }^{3} \mathrm{C}$ Charadva, ${ }^{3} \mathrm{~T}$ Morris,

${ }^{1 ;}{ }^{3}$ E Moss*. 'Leicester Cancer Research Centre, Leicester, UK; ${ }^{2}$ University of Leicester, Leicester Clinical Trials Unit, Leicester, UK; ${ }^{3}$ University Hospitals of Leicester, Gynaecological Oncology, Leicester, UK

\subsection{6/ijgc-2021-ESGO.145}

Introduction/Background* It is not known whether differences exist in the patient and endometrial cancer (EC) characteristics of South Asian patients currently living in England compared to women of White ethnicity.

Methodology A retrospective study of EC cases diagnosed at the University Hospitals of Leicester, UK between 2003-2018 was undertaken. Additional information on a subset of patients was available for patients recruited between January 2016 and January 2020.

Result(s)* A total of 1884 cases were included, 13\% of South Asian ethnicity. South Asian women were diagnosed at a significantly younger age, mean age 60.3 years, compared to women of White ethnicity, 66.9 years, mean difference $=6.6$ years (95\% CI 5.1 to 8.1), p $<0.001$. Rising BMI in the White ethnicity group significantly correlated with younger age at diagnosis $(\mathrm{p}<0.001)$, however this association was not seen in South Asian patients. Logistic regression analysis was performed. After adjusting for the diabetes status and BMI, South Asian patients were almost three time more likely to be diagnosed with EC below the age of 55 years, as compared to White ethnicity patients, odds ratio $=2.85(95 \% \mathrm{CI}$ 2.01 to 4.04$), \mathrm{p}<0.001$. Analysis of a subset of 216 cases (40 South Asian and 176 White ethnicity) identified that the number of South Asian patients who were pre-menopausal at diagnosis was more than double that in the White ethnicity group, 8 of 40 cases (20\%) compared to 16 of 176 cases $(9.1 \%),(p=0.048)$. For the patients who were postmenopausal there was no difference in the age of menopause, median age 51 years for both groups $(\mathrm{p}=0.408)$.

Conclusion* There are significant differences in the demographic characteristics between co-located South Asian and White ethnicity patients diagnosed with EC, in particular age at diagnosis and greater proportion of premenopausal cases seen in the South Asian ethnicity group. Further investigation is needed to explain these differences, including dietary and activity differences, and to determine their impact on suspected cancer referral criteria.

\section{CYTOREDUCTIVE SURGERY IN STAGE IV ENDOMETRIAL CANCER: A RETROSPECTIVE MULTICENTRE COHORT STUDY}

${ }^{1 ; 2}$ L Nooij, ${ }^{1} \mathrm{M}$ Uijterwaal, ${ }^{3} \mathrm{C}$ Lok, ${ }^{4} \mathrm{C}$ De Kroon, ${ }^{1} \mathrm{~J}$ Kasius, ${ }^{5} \mathrm{R}$ Zweemer, ${ }^{6} \mathrm{~N}$ Horeweg, ${ }^{7} \mathrm{~T}$ Bosse, ${ }^{5} \mathrm{~J}$ Van der Marel*. ${ }^{1}$ Amsterdam University Medical Center, Amsterdam, Department of Gynaecology, Amsterdam, Netherlands; '2 Leiden University Medical Center, Department of Gynaecology, Leiden, Netherlands; ${ }^{3}$ Antoni van Leeuwenhoek hospital, Department of Gynaecology, Amsterdam, Netherlands; ${ }^{4}$ Leiden University Medical Center, Department of Gynaecology, Leiden; ${ }^{5}$ University Medical Center Utrecht, Department of Gynaecology, Utrecht, Netherlands; ${ }^{6}$ Leiden University Medical Center (LUMC), Department of Radiation Oncology, Leiden, Netherlands; ' Leiden University Medical Center, Department of Pathology, Leiden, Netherlands

10.1136/ijgc-2021-ESG0.146 